\title{
Phase Behavior of Binary and Ternary Blends Having the Same Chemical Components and Compositions
}

\author{
Joung Eun Yoo, Yong Kim, and Chang Keun Kim* \\ Department of Chemical Engineering, Chung-Ang University, 221 Huksuk-Dong, Dongjak-Gu, Seoul 156-756, Korea
}

Jae Wook Lee

Department of Chemical Engineering, Sogang University, 1 Shinsoo-Dong, Mapo-Gu, Seoul 121-742, Korea

Received Jan. 13, 2003; Revised Sept. 1, 2003

\begin{abstract}
The phase behavior of binary blends of dimethylpolycarbonate-tetramethyl polycarbonate (DMPCTMPC) copolycarbonates and styrene-acrylonitrile (SAN) copolymers has been examined and then compared with that of DMPC/TMPC/SAN ternary blends having the same chemical components and compositions except that the DMPC and TMPC were present in the form of homopolymers. Both binary and ternary blends were miscible at certain blends compositions, and the miscible blends showed the LCST-type phase behavior or did not phase separated until thermal degradation temperature. The miscible region of binary blends is wider than that of the corresponding ternary blends. Furthermore, the phase-separation temperatures of miscible binary blends are higher than those of miscible ternary blends at the same chemical compositions. To explain the destabilization of polymer mixture with the increase of the number of component, interaction energies of binary pairs involved in these blends were calculated from the phase separation temperatures using lattice-fluid theory and then the phase stability conditions for the polymer mixture was analyzed with volume fluctuation thermodynamics.
\end{abstract}

Keywords: binary blends, ternary blends, LCST-type phase behavior, phase stability conditions, interaction energy.

\section{Introduction}

Successful design of polymer blend systems requires the ability to control or manipulate the phase behavior of the mixture. When homopolymer/homopolymer blend is not immiscible, a new miscible blend involving immiscible binary pair often produced via two different routes; Blending of a homopolymer with copolymer that composed of binary pair having strong intramolecular repulsion is known a useful route to prepare a miscible blend..$^{1-8}$ The other way preparing miscible blend is the homogenization of two immiscible polymers by adding a third polymer that is miscible with each component. ${ }^{9-17}$ According to the binary interaction model, ${ }^{1-3}$ the former method might be a useful route in developing miscible blends. In the previous researches, various miscible blends have been developed via copolymerization. ${ }^{4-8}$ The latter method also often used to develop a new miscible blend. However, this method was not successful in developing a new miscible blend unless the third component that is miscible with each component of blend is major

*e-mail : ckkim@cau.ac.kr

1598-5032/10/303-08@ 2003 Polymer Society of Korea component in blend. ${ }^{9-17}$

In this study, we have explored why the latter method could not be useful route in developing miscible blend in contrast with the former method. Binary blends and ternary blends composed of tetramethyl bisphenol-A polycarbonate (TMPC), dimethyl bisphenol-A polycarbonate (DMPC), and poly(styrene-co-acrylonitrile) copolymer (SAN) were explored as a sample system. To prepare binary blend containing three polymers, the synthesized DMPC-TMPC copolycarbonates were blended with SAN copolymers. Ternary blends having the same chemical component and composition with binary blends also prepared to compare their miscibility with that of binary blend. The quantitative information about interaction energies of binary pairs involved in blends is required in understanding phase behavior of blends. Interaction energies of binary pairs involved in the binary blends and ternary blends were calculated from the phase separation temperatures using the lattice-fluid theory ${ }^{18-23}$ combined with binary interaction model and then the phase stability of polymer mixture was analyzed with volume fluctuation thermodynamics. $^{15,18}$ 


\section{Experimental}

DMPC, TMPC, and DMPC-TMPC copolycarbonates were synthesized in our laboratory. Bisphenol monomers were reacted with bis(trichloromethyl) carbonate, triphosgen, to produce the polycarbonates using an interfacial polymerization technique. ${ }^{24}$ The interfacial polymerization used triethylamine as a phase transfer agent to transport salts from the aqueous phase into the organic phase where reaction with the soluble organic species occurs. The resulting polycarbonates dissolved in methylene chloride, were precipitated with methanol. Some of the SAN copolymers were obtained from external sources while remainder were synthesized. The synthesis of SAN copolymer was performed in bulk at $70^{\circ} \mathrm{C}$ with AIBN as an initiator and tri-dodecyl mercaptan as a chain transfer agent. Conversion for the synthesis of copolymers was kept about $10 \%$ to avoid composition drift. Molecular weight information of copolymers was obtained using GPC calibrated with polystyrene standards. The monomer content of the copolymers was determined by element analysis and H-NMR. Homopolymers and copolymers used in this study were listed in Table I. The numerical part of the code for copolymers indicates the weight percent of dimethylbisphenol-A monomer or that of AN.

Blends were prepared via solution casting from methylene chloride. The casting solutions were at $35^{\circ} \mathrm{C}$ for a day in an air circulating oven until most of the solvent had evaporated, and then the resulting films were further dried in a vacuum oven for a week. Glass transition temperatures was examined a differential scanning calorimeter (DSC, TA instrument, model DSC-2010) at a scanning rate of $20^{\circ} \mathrm{C} / \mathrm{min}$. The temperature at which phase separation caused by lower critical solution temperature, LCST, was measured by an annealing technique to access the closest true equilibrium temperature. ${ }^{6,25-27}$

\section{Results and Discussion}

Phase Behavior of Binary Blends. It was known that TMPC/PS blends and TMPC blends with SAN copolymers containing limited amounts of AN exhibit one-phase behavior. ${ }^{4-8}$ TMPC blends with SAN copolymers containing modest amounts of AN show higher phase separation temperatures (LCST-type) than TMPC/PS system. However, DMPC/SAN blends were found to be immiscible regardless of AN content of SAN copolymers. When miscible blends are obtained, the interaction energy can be quantified if the mixture shows phase separation upon heating or cooling. Since DMPC/PS and DMPC/SAN blends were immiscible, DMPC-TMPC copolymers were prepared at various compositions to obtain miscible blends with SAN copolymers.

When the copolycarbonate contains $40 \mathrm{wt} \%$ or less DMPC, blends with PS were miscible. On the other hand, blends of PS with copolycarbonates containing 50\% DMPC or more were cloudy at the casting condition and exhibited two glass transitions. Figure 1(a) shows the observed phase separation temperatures of miscible copolycarbonate/PS blends caused by LCST-type phase behavior. The effects of TMPC content

Table I. Polymers Used in This Study

\begin{tabular}{|c|c|c|c|c|}
\hline Polymer & Copolymer Composition $^{a}$ & ${\overline{M_{w}}}^{b}$ & $\overline{\bar{M}_{w}} /{\overline{M_{n}}}^{b}$ & Source \\
\hline PS & & 192,400 & 2.16 & LG Chemical \\
\hline SAN2 & $2 \% \mathrm{AN}$ & 213,000 & 1.72 & Asahi Chemical \\
\hline SAN5.7 & $5.7 \% \mathrm{AN}$ & 210,000 & 1.92 & Asahi Chemical \\
\hline SAN10 & $10 \% \mathrm{AN}$ & 215,000 & 2.03 & synthesized \\
\hline SAN15 & $15 \% \mathrm{AN}$ & 103,500 & 2.03 & synthesized \\
\hline SAN18 & $18 \% \mathrm{AN}$ & 13,500 & 1.95 & synthesized \\
\hline SAN20 & $20 \% \mathrm{AN}$ & 174,500 & 2.21 & synthesized \\
\hline SAN24 & $24 \% \mathrm{AN}$ & 153,000 & 1.83 & LG Chemical \\
\hline DMPC & & 48,300 & 2.47 & synthesized \\
\hline TMPC & & 46,700 & 2.07 & synthesized \\
\hline DMPC-TMPC 59 & $59 \mathrm{wt} \%$ DMPC & 40,500 & 2.05 & synthesized \\
\hline DMPC-TMPC 50 & $50 \mathrm{wt} \%$ DMPC & 30,500 & 1.79 & synthesized \\
\hline DMPC-TMPC 40 & $40 \mathrm{wt} \%$ DMPC & 23,400 & 1.69 & synthesized \\
\hline DMPC-TMPC 26 & $26 \mathrm{wt} \%$ DMPC & 29,100 & 1.56 & synthesized \\
\hline DMPC-TMPC 17 & $17 \mathrm{wt} \%$ DMPC & 28,400 & 1.64 & synthesized \\
\hline
\end{tabular}

${ }^{a}$ Monomer content in copolymer was determined by elemental analysis.

${ }^{b}$ Molecular weights were determined by GPC using polystyrene standards. 

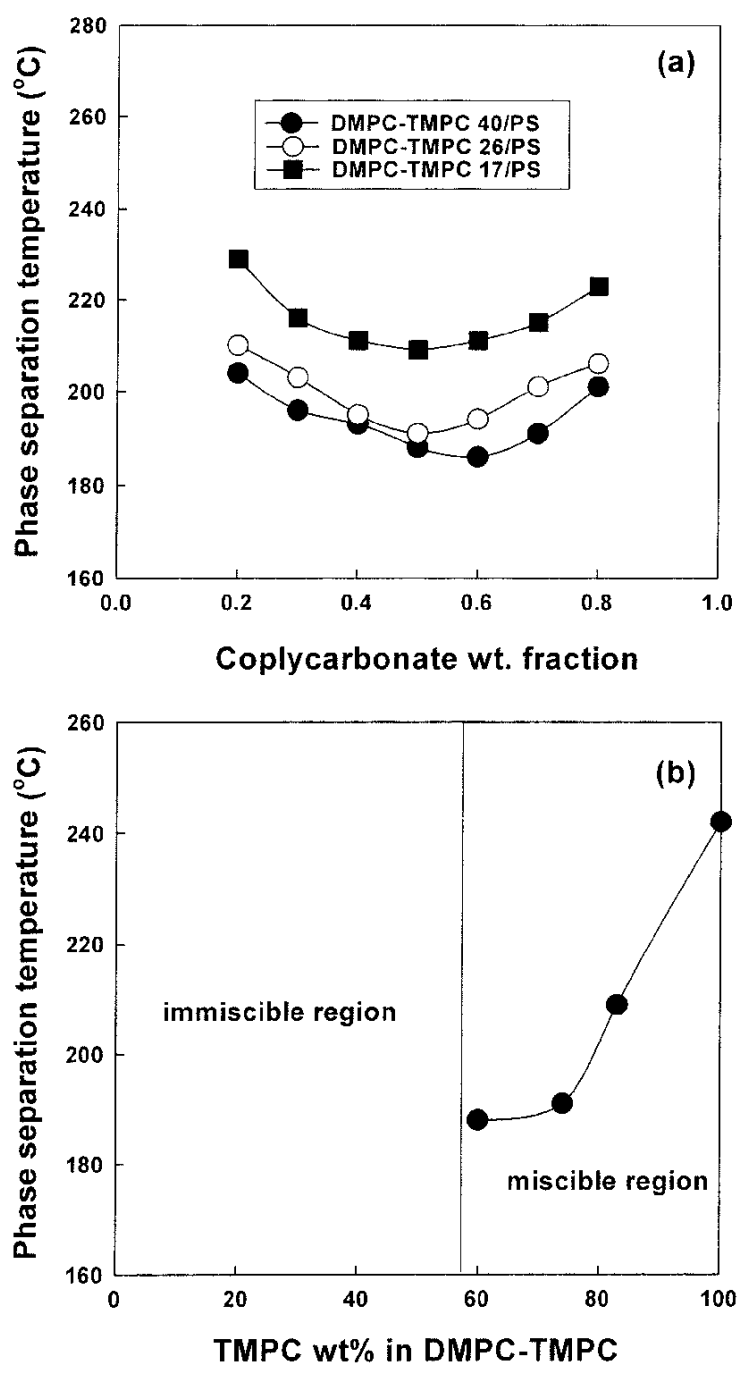

Figure 1. (a) Phase separation temperatures of PS blends with various DMPC-TMPC copolycarbonates. (b) Effects of copolycarbonate composition on the phase separation temperatures for 50/50=DMPC-TMPC/PS blends.

were exhibited in Figure 1(b) by plotting the phase separation temperature for a fixed PS content of blend (50 wt $\%$ ) versus the TMPC content of the copolycarbonates. The miscibility of these copolycarbonates with PS decreases as the DMPC content increases as expected. DMPC is miscible with TMPC but is immiscible with PS. ${ }^{4-8,24}$ According to the binary interaction model, the unfavorable interaction of DMPC with PS and the favorable interaction of DMPC and TMPC both act against miscibility of the copolycarbonate with PS.

When copolycarbonates contain $50 \mathrm{wt} \%$ DMPC or more, binary blends of DMPC-TMPC and SAN were not miscible regardless of the AN contents of SAN copolymers. However, copolycarbonates containing $40 \mathrm{wt} \%$ DMPC or less were formed miscible blends with SAN copolymers containing limited amounts of AN. DMPC-TMPC 40 copolycarbonates were miscible with SAN copolymers containing $10 \mathrm{wt} \% \mathrm{AN}$

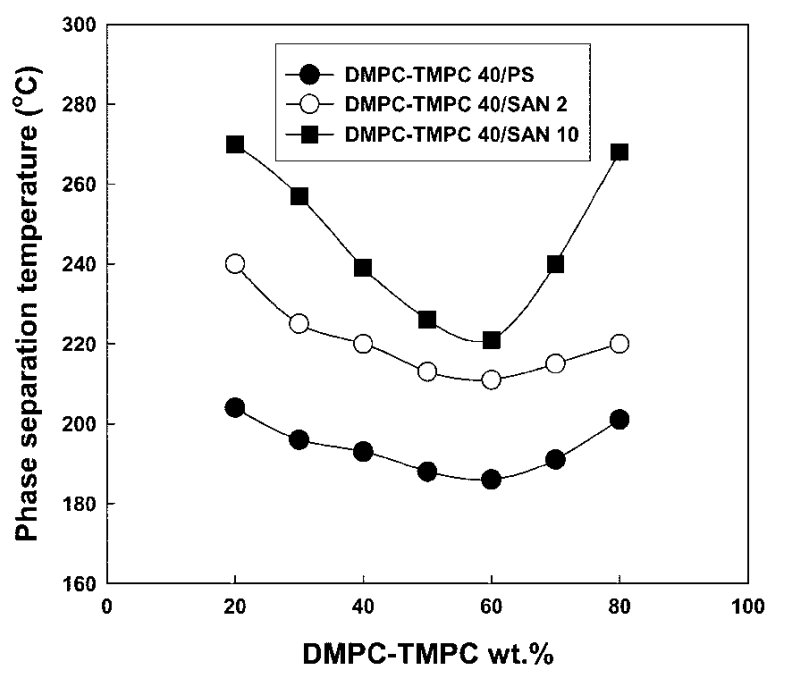

Figure 2. Phase separation temperatures of DMPC-TMPC 40 blends with SAN copolymers.

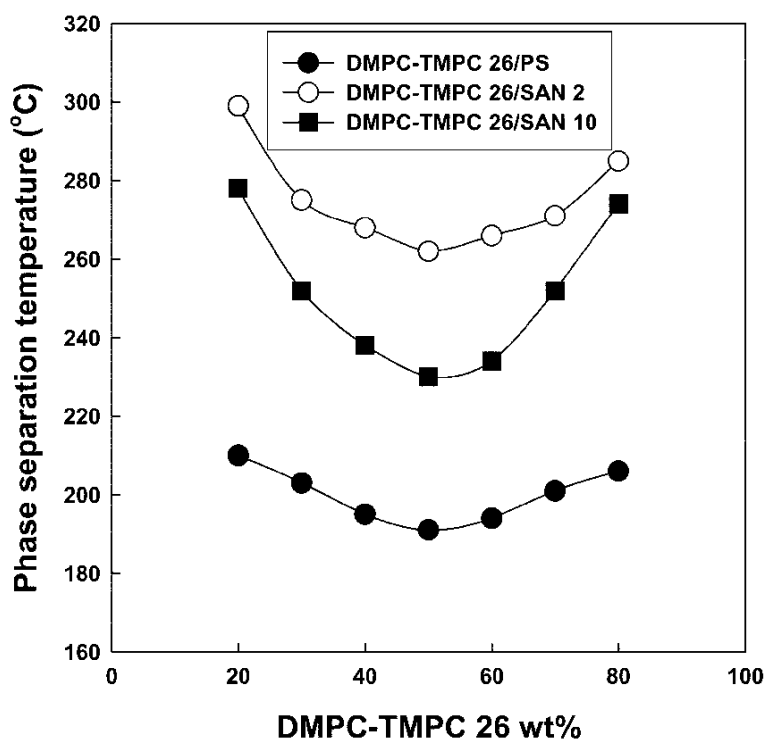

Figure 3. Phase separation temperatures of DMPC-TMPC 26 blends with SAN copolymers.

or less. Some of the miscible blends observed here exhibited LCST-type phase behavior, as shown in Figure 2. The phase separation curves are all very similar with each other showing a minimum at about $60 \mathrm{wt} \%$ DMPC-TMPC 40. Note that DMPC-TMPC 40 blends with SAN 5.7 copolymer did not phase separate on heating until thermal degradation temperature $\left(\sim 330^{\circ} \mathrm{C}\right)$. These results indicate that addition of $\mathrm{AN}$ to the styrene initially increases miscibility with DMPCTMPC but ultimately leads to immiscibility.

DMPC-TMPC 26/SAN blends were also miscible when SAN copolymers contain $10 \mathrm{wt} \%$ AN or less (Figure 3). DMPC-TMPC 26/SAN blends exhibited higher phase sepa- 


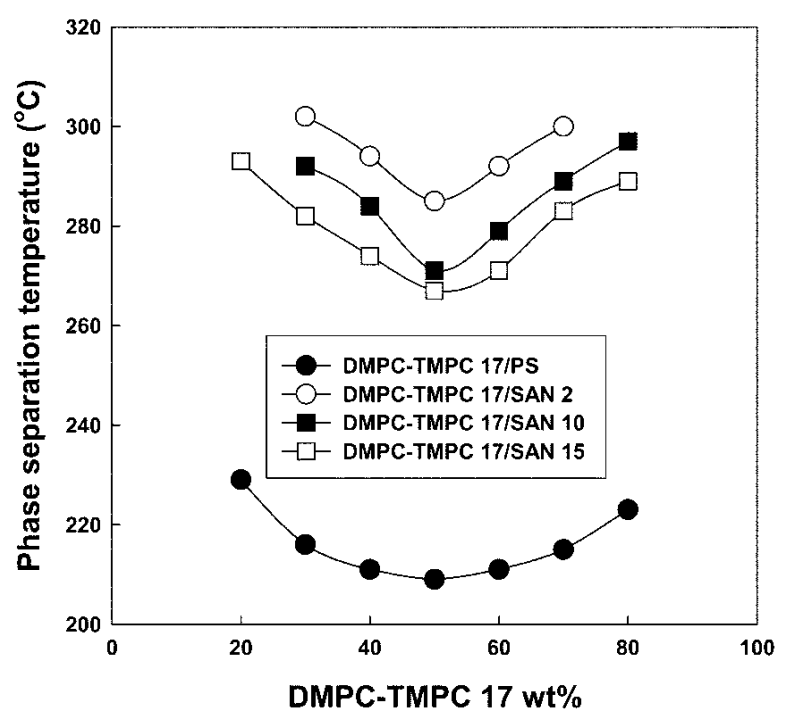

Figure 4. Phase separation temperatures of DMPC-TMPC 17 blends with SAN copolymers.

ration temperature than DMPC-TMPC 40/SAN blends when SAN copolymer contains the same amounts of AN. It means that the decline of DMPC content in copolycarbonates is favorable for the miscibility with SAN. DMPC-TMPC 17/ SAN blends were miscible when SAN copolymers contain less than or equal to $15 \mathrm{wt} \%$ AN (Figure 4). As shown in Figures 1-4, the decline of DMPC content in copolycarbonates broadens the miscible region of DMPC-TMPC/SAN blends and increases their phase separation temperatures. Note that TMPC is miscible with SAN copolymers containing $18 \mathrm{wt} \% \mathrm{AN}^{\text {? }}$

Phase Behavior of Ternary Blends. Phase behavior of ternary blends composed of DMPC, TMPC, and SAN were examined. DMPC-TMPC 40 copolymer formed miscible blends with SAN copolymers containing less than $10 \mathrm{wt} \%$ AN. However, DMPC/TMPC/SAN ternary blends having the same chemical compositions and components with DMPCTMPC 40/SAN binary blends except that the DMPC and TMPC were present in the form of homopolymers were not miscible with SAN copolymers. DMPC-TMPC 26 copolymer formed miscible blends with SAN copolymers containing less than or equal to $10 \mathrm{wt} \% \mathrm{AN}$ as mentioned before, while the corresponding DMPC/TMPC/SAN ternary blends were also miscible when SAN copolymers containing less than or equal to $10 \mathrm{wt} \%$ AN. As shown in Figure 5 for the selected DMPC-TMPC 26/ SAN = 5/5 blends and the corresponding ternary blends, phase separation temperature of binary blend is higher than that of the corresponding ternary blend.

To compare clearly phase behavior of binary blend with that of corresponding ternary blends, the miscibility map and experimentally determined phase separation temperatures for binary blends (50/50 composition) and those for corresponding ternary blends were shown in Figure 6. The miscible
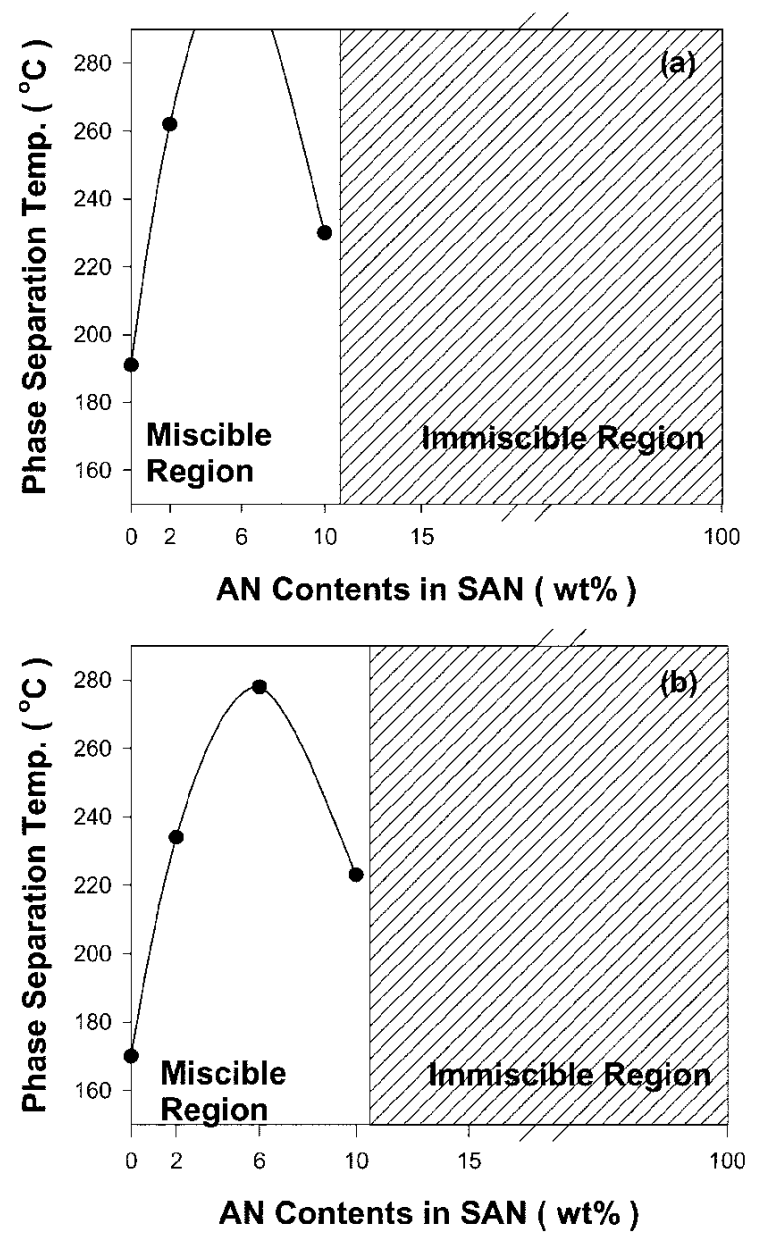

Figure 5. Miscibility windows of (a) 50/50 = DMPC-TMPC 26/ SAN binary blends and (b) the corresponding ternary blends, i.e., $50 / 50=(\mathrm{DMPC} / \mathrm{TMPC}=26 / 73) / \mathrm{SAN}$.

region of binary blend is broader than that of ternary blend and the phase separation temperature of binary blend is higher than that of the corresponding ternary blend. The results indicate that ternary blend is always less stable than binary blend having the same chemical compositions and components. To understand the phase behavior of blends, interaction energies of binary pairs involved in the blends were calculated from the phase separation temperatures using lattice-fluid theory combined with binary interaction model and then the phase stability conditions were discussed in the next section.

Interaction Energies and Phase Stability. The detail background calculating the interaction energies involved in the miscible blends from the phase boundaries using the lattice-fluid theory combined with binary and interaction model was described in the previous papers. ${ }^{6-8,18-27}$ The temperature at which phase separation caused by lower critical solution temperature, LCST, occurred, was measured by an annealing technique to access the closest true equilibrium temperature. The equation-of-state theory was adopted to avoid empirical 

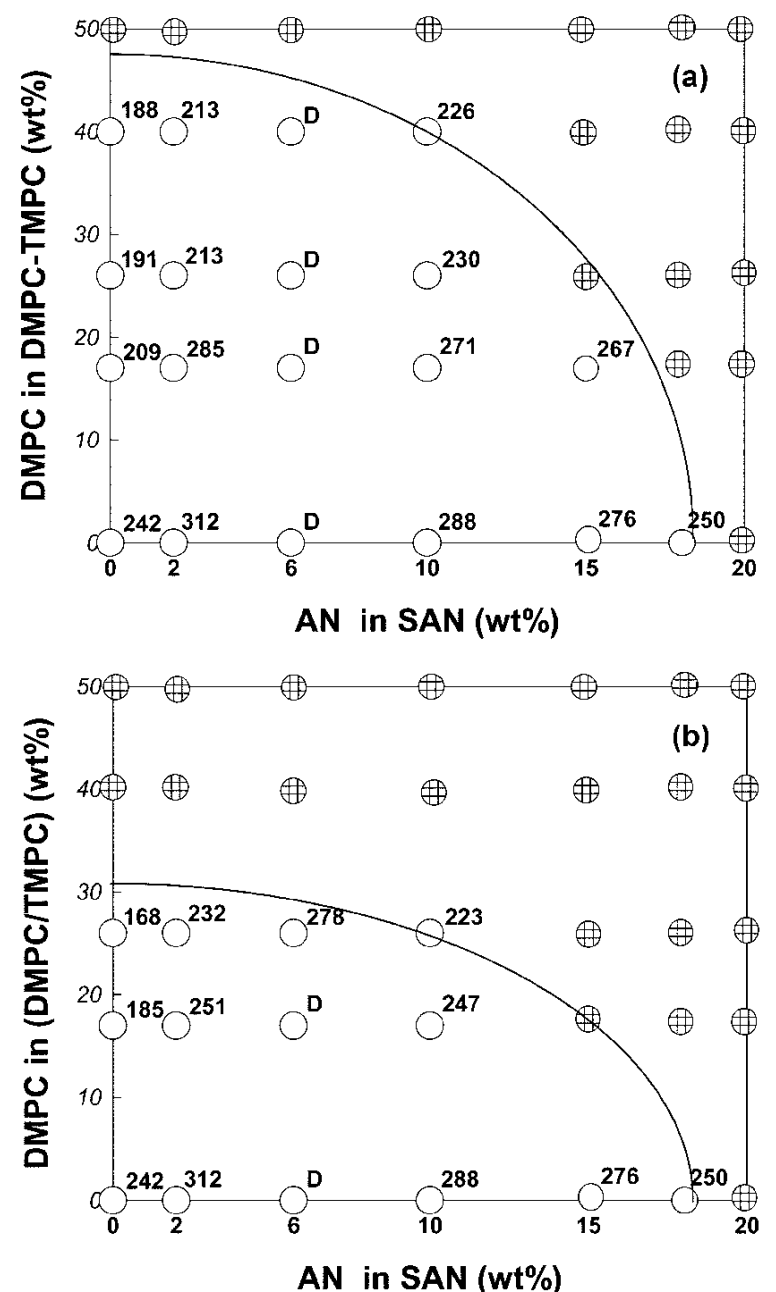

Figure 6. Miscibility maps for (a) 50/50 = DMPC-TMPC/SAN binary blends and (b) the corresponding ternary blends. Numbers indicate phase separation temperatures of blends and " $\mathrm{D}$ " means phase separation did not occurred until thermal degradation. Note that solid curves are spinodal curves.

expression in the modified Flory-Huggins theory and to clarify the reason for the LCST-type phase behavior. To extract information about interaction energy from the liquidliquid phase boundaries, it is assumed that to a good approximation these data correspond to the spinodal curve. ${ }^{6-8,25-27}$ The morphology of blend observed at a reported phase separation temperature could be also characterized by a high level of phase interconnectivity in both the minor and major phase caused by spinodal decomposition. According to the lattice fluid theory, the spinodal condition for a compressible binary mixture can be written

$$
\delta^{2} g=\left|\begin{array}{ll}
g_{11} & g_{\tilde{p} 1} \\
g_{\tilde{p} 1} & g_{\tilde{p} \tilde{p}}
\end{array}\right|=g_{11}-\frac{\left(g_{\tilde{p} 1}\right)^{2}}{g_{\tilde{p} \tilde{p}}}=0
$$

where $g$ is the free energy of mixing per unit volume and the subscripts 1 and $\tilde{p}$ indicate partial derivatives with respect to $\phi_{1}$ (volume fraction) or $\tilde{p}$ (reduced density). In terms of the Sanchez-Lacombe theory, the indicated derivatives for binary mixture are given by

$$
\begin{gathered}
g_{11}=-2 \tilde{\rho} \Delta P^{*}(i, j)+R T\left(\frac{1}{\phi_{1} r_{1} v_{1}{ }^{*}}+\frac{1}{\phi_{2} r_{2} v_{2}{ }^{*}}\right) \\
g_{\tilde{p}_{1}}=-\left(p_{1}^{*}-p_{2}^{*}-\left(1-2 \phi_{1}\right) \Delta P^{*}(i, j)\right)+\frac{R T}{\tilde{\rho}}\left(\frac{1}{r_{1} v_{1}{ }^{*}}-\frac{1}{r_{2} v_{2}{ }^{*}}\right) \\
-R T\left(\frac{1}{v_{1}^{*}}-\frac{1}{v_{2}^{*}}\right)\left(\frac{\ln (1-\tilde{\rho})}{\tilde{\rho}^{2}}+\frac{1}{\tilde{\rho}}\right) \\
g_{\tilde{p} \tilde{p}}=\frac{R T}{v^{*}}\left(\frac{2 \ln (1-\tilde{\rho})}{\tilde{\rho}^{3}}+\frac{1}{\tilde{\rho}^{2}(1-\tilde{\rho})}+\frac{1}{\tilde{\rho}^{2}}\left(1-\frac{1}{r}\right)\right)
\end{gathered}
$$

According to the binary interaction model, the interaction energy density between the pair of multicomponent polymers $i$ and $j$, i.e., $\Delta P^{*}(i, j)$ can be expressed as

$$
\Delta P^{*}(i, j)=\sum \sum_{k>l} \Delta P_{k l}^{*}\left(\phi_{k i}-\phi_{k j}\right)\left(\phi_{l j}-\phi_{l i}\right)
$$

In the above, $\Delta P_{k l}$ is the binary interaction energy density between units $k$ and $l$ while $\phi_{k i}$ is the volume fraction of $k$ unit in the polymer $i$. For a binary blend of copolymer $i$ composed of units 1 (styrene) and 2 (AN) with another copolymer $j$ composed of units 3 (DMPC) and 4 (TMPC), the interaction energy density between polymer $i$ and $j$ is given by

$$
\begin{aligned}
\Delta P^{*}(i, j) & =\Delta P_{13}^{*} \phi_{1}{ }^{\prime} \phi_{3}{ }^{\prime \prime}+\Delta P_{14}^{*} \phi_{1}{ }^{\prime} \phi_{4}{ }^{\prime \prime}+\Delta P_{23}^{*} \phi_{2}{ }^{\prime} \phi_{3}{ }^{\prime \prime} \\
& +\Delta P_{24}^{*} \phi_{2}{ }^{\prime} \phi_{4}{ }^{\prime}-\Delta P_{12}^{*} \phi_{1}{ }^{\prime} \phi_{2}{ }^{\prime \prime}-\Delta P_{34}^{*} \phi_{3}{ }^{\prime} \phi_{4}{ }^{\prime \prime}
\end{aligned}
$$

where $\phi_{k}^{\prime}$ and $\phi^{\prime \prime}{ }_{k}$ denote volume fraction of unit $k$ in copolymer $i$ and $j$.

The characteristic parameters of each polymer evaluated from pressure-volume-temperature (PVT) data in the previ-

Table II. Characteristic Properties of Polymers

\begin{tabular}{lcccc}
\hline Polymers & $T^{*}(\mathrm{~K})$ & $P^{*}(\mathrm{bar})$ & $\rho^{*}\left(\mathrm{gcm}^{-3}\right)$ & References \\
\hline PS & 810 & 3,725 & 1.0922 & {$[28]$} \\
SAN2 & 803 & 3,777 & 1.0935 & {$[7]$} \\
SAN5.7 & 815 & 3,792 & 1.0982 & {$[7]$} \\
SAN10 & 819 & 3,834 & 1.1028 & {$[7]$} \\
SAN15 & 823 & 3,893 & 1.1081 & {$[7]$} \\
SAN20 & 828 & 3,949 & 1.1135 & {$[7]$} \\
SAN24 & 832 & 4,001 & 1.1179 & {$[7]$} \\
DMPC & 781 & 4,742 & 1.1830 & {$[25]$} \\
TMPC & 729 & 4,395 & 1.1854 & {$[28]$} \\
\hline
\end{tabular}




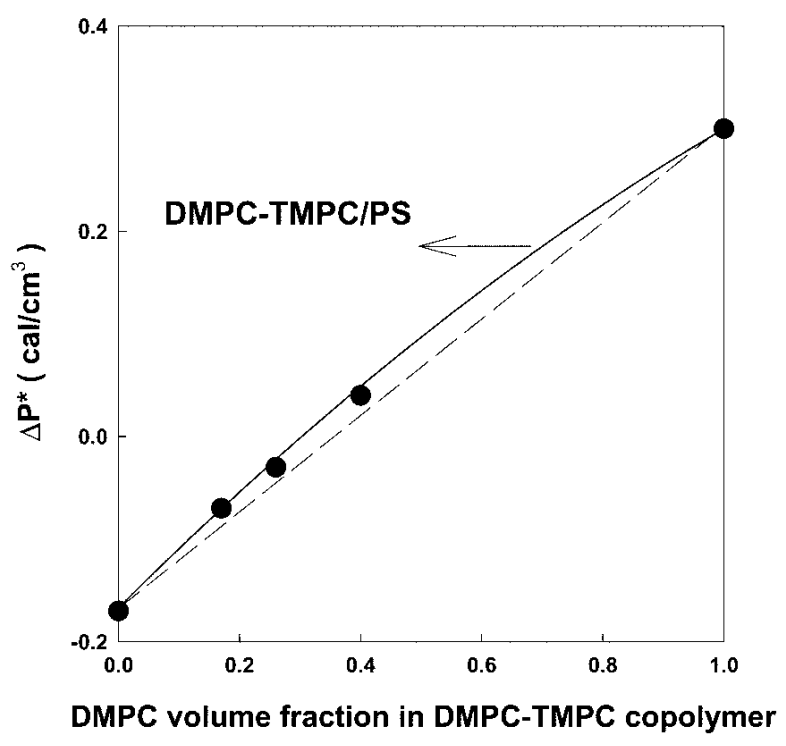

Figure 7. Interaction energies for blends of DMPC-TMPC with PS. Note that the solid line was obtained by fitting the interaction energies to equation (5) using linear regression.

ous researches ${ }^{7-8,25}$ were listed in Table II. As shown in Figure 7 , the calculated $\Delta P^{*}(i, j)$ for each DMPC-TMPC with PS using the theoretical spinodal condition, i.e., equation (1) is negative and monotonically increases with DMPC content. It means that DMPC units in copolycarbonates are unfavorable for miscibility with PS. According to the binary interaction model, the up-convex curvature stems from the favorable interaction between DMPC and TMPC. Using $\Delta P_{T M P C-S}^{*}=$ $-0.17 \mathrm{cal} / \mathrm{cm}^{3}$ obtained in the previous research ${ }^{28}$ from the phase behavior of TMPC/PS blends, the interaction energy densities for the remaining monomer unit pairs in DMPCTMPC/PS system were obtained by fitting the calculated values to equation (6) using linear regression. The calculated interaction energy densities for DMPC-PS and DMPCTMPC pairs, i.e., $\Delta P^{*}{ }_{D M P C-S}$ and $\Delta P^{*}{ }_{D M P C-T M P C}$ were $0.30 \mathrm{cal} /$ $\mathrm{cm}^{3}$ and $-0.09 \mathrm{cal} / \mathrm{cm}^{3}$, respectively. Using the interaction energy densities obtained here and those determined previously, ${ }^{7-9}$ i.e., $\Delta P_{S-A N}^{*}=7.37 \mathrm{cal} / \mathrm{cm}^{3}, \Delta P^{*}{ }_{T M P C-S}=-0.17 \mathrm{cal} / \mathrm{cm}^{3}$, $\Delta P_{T M P C-A N}^{*}=5.92 \mathrm{cal} / \mathrm{cm}^{3}, \quad \Delta P_{D M P C-S}^{*}=0.30 \mathrm{cal} / \mathrm{cm}^{3}$, and $\Delta P_{D M P C-T M P C}^{*}=-0.09 \mathrm{cal} / \mathrm{cm}^{3}$, the interaction energy density for the remai-ning monomer unit pair for DMPC-TMPC/ SAN system, i.e., $\triangle P_{D M P C-A N}^{*}$ obtained in a similar fashion was $4.60 \mathrm{cal} / \mathrm{cm}^{3}$.

The interaction energy densities of the binary pairs involving in the various polycarbonates and SAN were listed in Table III. To examine the reliability of the interaction energy densities obtained here, the spinodal curve calculated from the equation (1) and values listed in Table III. Figure 8 showed that experimental phase separation temperatures and the spinodal curve calculated from the equation (1) with a fixed value of $\triangle P^{*}{ }_{D M P C-T M P C 26-S A N 10}=-0.12 \mathrm{cal} / \mathrm{cm}^{3}$ for
Table III. Interaction Energies of Various Binary Pairs

\begin{tabular}{lccl}
\hline \multicolumn{1}{c}{$\begin{array}{c}\text { Interaction } \\
\text { Pairs }\end{array}$} & $\begin{array}{c}\Delta P^{*}\left(\mathrm{cal} / \mathrm{cm}^{3}\right) \\
(\text { This Study) }\end{array}$ & $\begin{array}{c}\Delta P^{*}\left(\mathrm{cal} / \mathrm{cm}^{3}\right) \\
(\text { Previous Studies })\end{array}$ & References \\
\hline PC-S & - & 0.43 & {$[8,26]$} \\
DMPC-S & 0.3 & 0.3 & {$[25]$} \\
TMPC-S & - & -0.17 & {$[28]$} \\
PC-AN & - & 4.4 & {$[8]$} \\
DMPC-AN & 4.6 & - & \\
TMPC-AN & - & 5.92 & {$[7]$} \\
PC-TMPC & - & -0.23 & {$[8]$} \\
DMPC-TMPC & -0.09 & - & \\
\hline
\end{tabular}

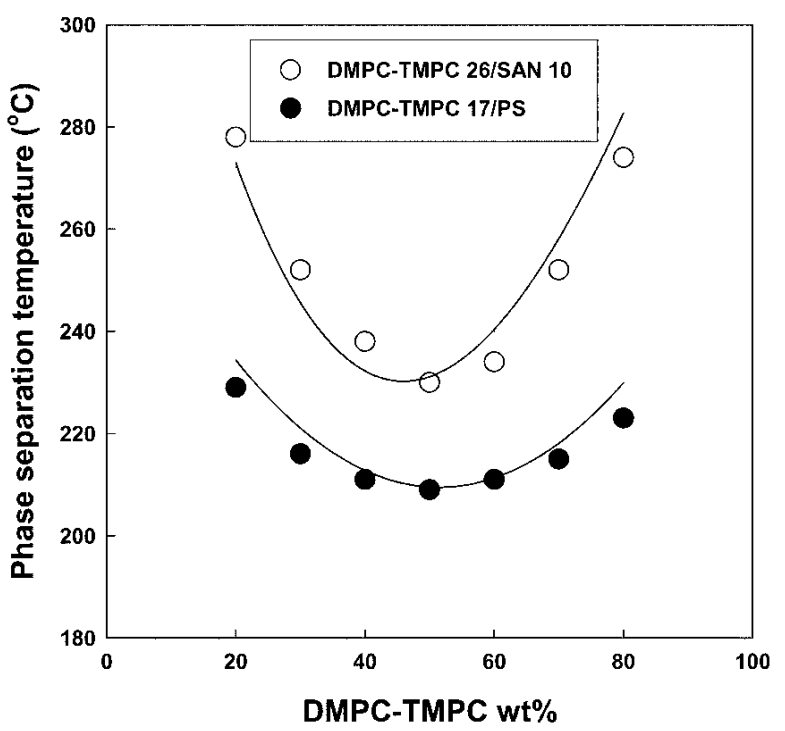

Figure 8. Phase separation temperatures of the selected DMPCTMPC/SAN and DMPC-TMPC/PS blends. Note that the spinodal curves were calculated from the equation (1) with a fixed value of $\Delta P^{*}{ }_{D M P C-T M C 26-S A N 10}=-0.12 \mathrm{cal} / \mathrm{cm}^{3}$ for DMPC-TMPC 26/SAN 10 blends and with $\Delta P^{*}{ }_{D M P C-T M C 17 / P S}=-0.07 \mathrm{cal} / \mathrm{cm}^{3}$ for DMPC-TMPC 17/PS.

DMPC-TMPC 26/SAN 10 blends and with $\triangle P^{*}{ }_{D M P C-T M P C 17 / P S}$ $=-0.07 \mathrm{cal} / \mathrm{cm}^{3}$ for DMPC-TMPC 17/PS. The curves agree with the experimentally determined phase separation temperatures.

According to the binary interaction model, the free energy term related to the interaction energy for binary blend and ternary blend is given by

$$
\begin{aligned}
& \left(\Delta g_{n c}\right)_{\text {binary }}=\Delta P_{13}^{*} \phi_{1} \phi_{3}+\Delta P_{14}^{*} \phi_{1} \phi_{4}+\Delta P_{23}^{*} \phi_{2} \phi_{3} \\
& +\Delta P_{24}^{*} \phi_{2} \phi_{4}-\Delta P_{12}^{*} \phi_{1} \phi_{2}^{\prime}\left(\phi_{3}+\phi_{4}\right)-\Delta P_{34}^{*} \phi_{3} \phi_{4}^{\prime \prime}{ }_{4}\left(\phi_{1}+\phi_{2}\right) \\
& \left(\Delta g_{n c}\right)_{\text {ternary }}=\Delta P_{13}^{*} \phi_{1} \phi_{3}+\Delta P_{14}^{*} \phi_{1} \phi_{4}+\Delta P_{23}^{*} \phi_{2} \phi_{3} \\
& +\Delta P_{24}^{*} \phi_{2} \phi_{4}-\Delta P_{12}^{*} \phi_{1} \phi_{2}^{\prime}\left(\phi_{3}+\phi_{4}\right)+\Delta P_{34}^{*} \phi_{3} \phi_{4}
\end{aligned}
$$


The difference between these two is given by

$$
\left(\Delta g_{n c}\right)_{\text {ternary }}-\left(\Delta g_{n c}\right)_{\text {binary }}=\Delta P_{34}^{*} \phi_{3}\left[\phi_{4}+\phi^{\prime \prime}{ }_{4}\left(\phi_{1}+\phi_{2}\right)\right]
$$

where $\phi_{k}$ is volume fraction of component $k$ in blend, and $\phi_{k}^{\prime}$ and $\phi^{\prime \prime}{ }_{k}$ denote volume fraction of unit $k$ in copolymer $i$ and $j$. Note that 1, 2, 3, and 4 indicate styrene, acrylonitrile, TMPC, and DMPC, respectively. Since the interaction energy between TMPC and DMPC is negative, i.e. $\Delta P_{34}^{*}<0$, the term related interaction energy for the ternary blend is always smaller than that for the binary blend. It is generally expected that the greater interaction energy a mixture has, the more difficult it is to achieve phase stability. Even though interaction energy of the ternary blend is more favorable than that of binary blend, the region where ternary blends are miscible is much narrower that that of binary blends. In addition, phase separation temperatures of the miscible ternary blends are lower than those of the corresponding binary blends. To explain this abnormal observation, phase stability conditions of ternary blend examined. ${ }^{15,18}$ According to the volume fluctuation thermodynamics, ternary blend is miscible when the following conditions are satisfied

$$
\delta^{2} g=\left|\begin{array}{lll}
g_{11} & g_{12} & g_{1 \tilde{\rho}} \\
g_{21} & g_{22} & g_{2 \tilde{\rho}} \\
g_{31} & g_{3 \tilde{\rho}} & g_{\tilde{\rho} \tilde{\rho}}
\end{array}\right|>0
$$

where the subscripts 1,2 , and $\tilde{\rho}$ indicate partial derivatives with respect to, $\phi_{1}, \phi_{2}$, or $\tilde{\rho}$. The solid curves in Figure 6 are the calculated spinodal curves for binary blends (Figure 6(a)) and ternary blends (Figure 6(b)). The spinodal curve satisfies $\delta^{2} g=0$. The miscible region of binary blends that satisfies $\delta^{2} g>0$ in equation (1) broader than that of ternary blends that satisfies $\delta^{2} g>0$ in equation (10). Binary blend is always miscible when its interaction energy is negative. However, some ternary blends that have negative interaction energy, i.e., the negative value of Gibbs free energy of mixing, are immiscible because these blends do not satisfy stability condition of equation (10). According to the volume fluctuation thermodynamics, ${ }^{15,18}$ it is known that the addition of component, accompanied by the asymmetry in the binary interactions, results in destabilization of blend.

\section{Summary}

The phase behavior of DMPC-TMPC/SAN binary blends and DMPC/TMPC/SAN ternary blends having the same chemical compositions and components has been explored theoretically and experimentally. The DMPC-TMPC 40/SAN binary blends formed miscible blends with SAN copolymers containing less than $10 \mathrm{wt} \% \mathrm{AN}$, while the ternary blends having the same chemical compositions did not form a miscible blend with SAN copolymers. Binary blends of DMPC-TMPC 26/SAN and DMPC-TMPC 17/SAN and the corresponding ternary blends were miscible when SAN copolymers contain limit amounts of AN. The miscible region of ternary blends was much narrower than that of binary blends. In addition, the phase-separation temperatures of miscible ternary blends are always lower than those of the corresponding binary blends. To explain the difference in the phase diagram of binary blends and ternary blends, phase stability conditions based on the volume fluctuation thermodynamics was explored with interaction energies of binary pairs obtained here. In the blends containing TMPC, DMPC, and SAN, even though the interaction energy of ternary blend is always more favorable for miscibility than that of binary blend, the additional degree of freedom, i.e., increase of the number of component, results in destabilization of blend.

Acknowledgements. This study was supported by research grants from the Korea Science and Engineering Foundation (KOSEF) through the Applied Rheology Center (ARC), an official KOSEF-created engineering research center (ERC) at Korea University, Seoul, Korea.

\section{References}

(1) D. R. Paul and J. W. Barlow Polymer, 25, 487, (1984).

(2) R. P. Kambour, T. J. Bendler, and R. C. Bopp, Macromolecules, 16, 1827, (1983).

(3) G. ten Brinke, F. E. Karasz, and W. J. Macknight, Macromolecules, 16, 1827, (1983).

(4) K. E. Min and D. R. Paul, Macromolecules, 20, 2828, (1987).

(5) A. C. Fernandes, J. W. Barlow, and D. R. Paul, Polymer, 27, 1789 (1986).

(6) M. J. Kim, J. E. Yoo, H. K. Choi, and C. K. Kim, Macromol. Res., 10, 91 (2002).

(7) C. K. Kim and D. R. Paul, Polymer, 33, 2089 (1992).

(8) C. K. Kim and D. R. Paul, Polymer, 33, 4929 (1992).

(9) T. K. Kwei, H. L. Frisch, W. Radigan, and S. Vogel, Macromolecules, 10, 157(1977).

(10) Y. Y. Wang and S. A. Chen, Polym. Eng. Sci., 21, 47 (1981).

(11) D. Rigby, J. L. Lin, and R. J. Roe, Macromolecules, 18, 2269 (1985).

(12) V. Shah, J. D. Keitz, D. R. Paul, and J. W. Barlow, J. Appl. Polym. Sci., 32, 3863 (1986).

(13) J. I. Equizabal, J. J. Irvin, M. Cotazar, and G. M. Guzman, J. Appl. Polym. Sci., 32, 5945 (1986).

(14) W. H. Christiansen, D. R. Paul, and J. W. Barlow, J. Appl. Polym. Sci., 34, 537 (1987).

(15) G. R. Brannock and D. R. Paul, Macromolcules, 23, 5240 (1990).

(16) C. J. T. Landry, H. Yang, and J. S. Machell, Polymer, 32, 44 (1991).

(17) J. A. Pomposo, E. Calahorra, I. Eguiazabal, and M. Cortazar, Macromolcules, 26, 2104 (1993).

(18) I. C. Sanchez, Macromolecules, 24, 908 (1991).

(19) I. C. Sanchez and A. C. Balaz, Macromolecules, 22, 2325 (1989). 
(20) I. C. Sanchez, Encyclopedia of Physical Science and Technology, Academic Press Academic Press, New York, 1987, Vol. XI, pp 1.

(21) I. C. Sanchez and R. H. Lacombe, J. Phys. Chem., 80, 2568 (1976).

(22) I. C. Sanchez and R. H. Lacombe, J. Phys. Chem., 80, 2358 (1976).

(23) I. C. Sanchez and R. H. Lacombe, Macromolecules, 11, 1145 (1978).
(24) C. K. Kim and D. R. Paul, Macromolecules, 25, 3097 (1992).

(25) J. H. Kim, D. S. Park, and C. K. Kim, J. Polym. Sci.: Polym. Phys. Ed., 38, 2666 (2000).

(26) T. A. Callaghan and D. R. Paul Macromolecules, 26, 2439, (1993).

(27) J. H. Kim, J. E. Yoo, and C. K. Kim, Macromol. Res., 10, 209 (2002).

(28) C. K. Kim and D. R. Paul, Polymer, 33, 1630 (1992). 